\title{
Cloud Computing: Overview \& Current Research Challenges
}

\author{
Mohsin Nazir \\ Department of Information Technology, Central University of Kashmir, India
}

\begin{abstract}
Cloud computing is a set of IT services that are provided to a customer over a network on a leased basis and with the ability to scale up or down their service requirements. Usually Cloud Computing services are delivered by a third party provider who owns the infrastructure.Cloud Computing holds the potential to eliminate the requirements for setting up of high-cost computing infrastructure for IT-based solutions and services that theindustry uses. It promises to provide a flexible IT architecture, accessible through internet from lightweight portable devices. This would allow multi-fold increase in the capacity and capabilities of the existing and new software.This new economic model for computing has found fertile ground and is attracting massive global investment. Many industries, such as banking, healthcare and education are moving towards the cloud due to the efficiency of services provided by the pay-per-use pattern based on the resources such as processing power used, transactions carried out, bandwidth consumed, data transferred, or storage space occupied etc. In a cloud computing environment, the entire data resides over a set of networked resources, enabling the data to be accessed through virtual machines. Despite the potential gains achieved from the cloud computing, the organizations are slow in accepting it due to security issues and challenges associated with it. Security is one of the major issues which hamper the growth of cloud. There are various research challenges also there for adopting cloud computing such as well managed service level agreement (SLA), privacy, interoperability and reliability.This research paper presents what cloud computing is, the various cloud models and the overview of the cloud computing architecture. This research paper also analyzes the key research challenges present in cloud computing and offers best practices to service providers as well as enterprises hoping to leverage cloud service to improve their bottom line in this severe economic climate.
\end{abstract}

Keywords:Cloud Architecture, Cloud Computing, Software as a Service (SaaS), Platform as a Service (PaaS),Infrastructure as a Service (IaaS), Mobile Cloud Computing (MCC).

\section{INTRODUCTION}

Internet has been a driving force towards the various technologies that have been developed. Arguably, one of the most discussed among all of these is Cloud Computing. Over the last few years, cloud computing paradigm has witnessed an enormous shift towards its adoption and it has become a trend in the information technology space as it promises significant cost reductions and new business potential to its users and providers [23]. The advantages of using cloud computing include:

i) reduced hardware and maintenance cost,

ii) accessibility around the globe, and

iii) flexibility and highly automated processes wherein the customer need not worry about mundane concerns like software up-gradation [15, 34].

Cloud Computing $[4,17]$ is an emerging trend to deploy and maintain software and is being adopted by the industry such as Google, IBM, Microsoft, and Amazon. Several prototype applications and platforms, such as the IBM - Blue Cloudinfrastructure, the Google App Engine, the Amazon Cloud, and the Elastic Computing Platform [42]. Cloud Computing is perceived as the next progression that will impact organizational businesses and how they manage their IT infrastructures. The technology and architecture that cloud service and deployment models offer are a key area of research.

Even though there are numerous variations on the definition of Cloud Computing, some basic principles characterize this emerging computing paradigm. Cloud Computing providestechnological capabilities - generally maintained off premises - that are delivered on demand as a service via the Internet. Given that a third party owns and manages public cloud services, consumers of these services do not possess resources in the cloud model but pay for them on a per-use basis.Thus virtualization of the resources is the key concept [37]. In the real scenario, they are renting the physical infrastructure, platforms and applications within a shared architecture. Cloud offerings can vary from virtual infrastructure, computing platforms, centralized data centers to end-user Web-Services and Web applications to enormous other focused computing services.

Cloud Computing may be applied to solve problems in manydomains of Information Technology like GIS (Geographical Information Systems), Scientific Research [7], e-GovernanceSystems [19], Decision Support Systems [42], ERP [17], Web Application Development [21], Mobile Technology [36] etc. 


\section{CLOUd COMPUTING: OVERVIEW}

According to U.S National Institute of Standards and Technology (NIST), "Cloud Computing is a model for enabling convenient, on-demand network access to a shared pool of configurable computing resources (e.g., networks, servers, storage, applications, and services) that can be rapidly provisioned and released with minimal management effort or cloud provider interaction" [27]. In simple words, Cloud Computing is the combination of a technology, platform that provides hosting and storage service on the Internet [14].In such an environment users need not own the infrastructure for various computing services. In fact, they can be accessed from any computer in any part of the world. This integrates features supporting high scalability and multitenancy, offering enhanced flexibility in comparison to the earlier existing computing methodologies. It can deploy, allocate or reallocate resources dynamically with an ability to continuously monitor their performance [27]. Moreover, cloud computing minimizes the capital expenditure. This approach is device and user-location independent. Main goal of the cloud computing is to provide scalable and inexpensive on-demand computing infrastructures with good quality of service levels $[9,18]$.

Cloud Computing is a general term for anything that involves delivering hosted services over the Internet. Instead of a static system architecture, Cloud Computing supports the ability to dynamically scale up and quickly scale down, offering cloud consumers high reliability, quick response times, and the flexibility to handle traffic fluctuations and demand [22]. CloudComputing also supports multi tenancy, providing systems configured in such a way that they can be pooled to be shared by many organizations or individuals [8]. Virtualization technology allows cloud vendors to convert one server into many virtual machines, thereby eliminating client-server computing with single-purpose systems [17]. This maximizes hardware capacity and allows customers to leverage economies of scale [22].

Benefits of Cloud computing are enormous. The most important one is that the customers don't need to buy the resource from a third party vendor, instead they can use the resource and pay for it as a service thus helping the customer to save time and money.Cloud is not only for Multinational companies but it's also being used by small and medium enterprises [30].

\section{A. DEPLOYMENT MODELS}

\section{CLOUd COMPUTING BUILDING BLOCKS}

In the cloud deployment model, networking, platform, storage, and software infrastructure are provided as services that scale up or down depending on the demand as depicted in figure 1. The Cloud Computing model has four main deployment models which are:

Private Cloud: Private cloud is a new term that some vendors have recently used to describe offerings that emulate cloud computing on private networks. It is set up within an organization's internal enterprise datacenter. In the private cloud, scalable resources and virtual applications provided by the cloud vendor are pooled together and available for cloud users to share and use. It differs from the public cloud in that all the cloud resources and applications are managed by the organization itself, similar to Intranet functionality. Utilization on the private cloud can be much more secure than that of the public cloud because of its specified internal exposure. Only the organization and designated stakeholders may have access to operate on a specific Private cloud [38]. One of the best examples of a private cloud is Eucalyptus Systems [3].

Public Cloud: Public cloud describes cloud computing in the traditional mainstream sense, whereby resources are dynamically provisioned on a fine-grained, self-service basis over the Internet, via web applications/web services, from an off-site third-party provider who shares resources and bills on a fine-grained utility computing basis. It is typically based on a pay-per-use model, similar to a prepaid electricity metering system which is flexible enough to cater for spikes in demand for cloud optimization [1]. Public clouds are less secure than the other cloud models because it places an additional burden of ensuring all applications and data accessed on the public cloud are not subjected to malicious attacks. Examples of a public cloud include Microsoft Azure, Google App Engine.

Hybrid Cloud: Hybrid cloud is a private cloud linked to one or more external cloud services, centrally managed, provisioned as a single unit, and circumscribed by a secure network [12]. It provides virtual IT solutions through a mix of both public and private clouds. Hybrid Cloud provides more secure control of the data and applications and allows various parties to access information over the Internet. It also has an open architecture that allows interfaces with other management systems. Hybrid cloud can describe configuration combining a local device, such as a Plug computer with cloud services. It can also describe configurations combining virtual and physical, collocated assets -for example, a mostly virtualized environment that requires physical servers, routers, or other hardware such as a network appliance acting as a firewall or spam filter. An example of a Hybrid Cloud includes Amazon Web Services (AWS). 
Community Cloud: Infrastructure shared by several organizations for a shared cause and may be managed by them or a third party service provider and rarely offered cloud model. These clouds are normally based on an agreement between related business organizations such as banking or educational organizations. A cloud environment operating according to this model may exist locally or remotely. An example of a Community Cloud includes Facebook.



Fig. 1 Cloud Deployment Model [1]

Moreover, with the technological advancements, we can see derivative cloud deployment models emerging out of the various demands and the requirements of users. A similar example being a virtual-private cloud wherein a public cloud is used in a private manner, connected to the internal resources of the customer's data-centre [40]. With the emergence of high-end network access technologies like 2G, 3G, Wi-Fi, Wi-Max etc. and feature phones, a new derivative of cloud computing has emerged. This is popularly referred as "Mobile Cloud Computing (MCC)". It can be defined as a composition of mobile technology and cloud computing infrastructure where data and the related processing will happen in the cloud only with an exception that they can be accessed through a mobile device and hence termed as mobile cloud computing [16]. It's becoming a trend now-a-days and many organizations are keen to provide accessibility to their employees to access office network through a mobile device from anywhere.

Recent technical advancements including the emergence of HTML5 and various other browser development tools have only increased the market for mobile cloud-computing. An increasing trend towards the feature-phone adoption [16] has also ramped up the MCC market.

\section{B. SERVICE MODELS}

According to the different types of services offered, cloud computing can be considered to consist of three layers: software as a service (SAAS), platform as a Service (PAAS), and infrastructure as a Service (IAAS) (Iyer and Henderson, 2010; Han, 2010, Mell and Grance, 2010). Infrastructure as a Service (IaaS) is the lowest layer that provides basic infrastructure support service. Platform as a Service (PaaS) layer is the middle layer, which offers platform oriented services, besides providing the environment for hosting user's applications. Software as a Service (SaaS) is the topmost layer which features a complete application offered as service on demand $[2,24]$.

Software-as-a-Service (SaaS):SaaS can be described as a process by which Application Service Provider (ASP) provide different software applications over the Internet. This makes the customer to get rid of installing and operating the application on own computer and also eliminates the tremendous load of software maintenance; continuing operation, safeguarding and support [33]. SaaS vendor advertently takes responsibility for deploying and managing the IT infrastructure (servers, operating system software, databases, data center space, network access, power and cooling, etc) and processes (infrastructure patches/upgrades, application patches/upgrades, backups, etc.) required to run and manage the full solution. SaaS features a complete application offered as a service on demand. In SaaS, there is the Divided Cloud and Convergence coherence mechanism whereby every data item has either the "Read Lock" or "Write Lock" [11]. Two types of servers are used by SaaS: the Main Consistence Server (MCS) and Domain Consistence Server (DCS). Cache coherence is achieved by the 
cooperation between MCS and DCS [35]. In SaaS, if the MCS is damaged, or compromised, the control over the cloud environment is lost. Hence securing the MCS is of great importance.Examples of SaaS includes: Salesforce.com, Google Apps.

Platform as a Service (PaaS): "PaaS is the delivery of a computing platform and solution stack as a service without software downloads or installation for developers, IT managers or end-users. It provides an infrastructure with a high level of integration in order to implement and test cloud applications. The user does not manage the infrastructure (including network, servers, operating systems and storage), but he controls deployed applications and, possibly, their configurations. Examples of PaaS includes: Force.com, Google App Engine and Microsoft Azure.

Infrastructure as a Service (IaaS): Infrastructure as a service (IaaS) refers to the sharing of hardware resources for executing services using Virtualization technology. Its main objective is to make resources such as servers, network and storage more readily accessible by applications and operating systems. Thus, it offers basic infrastructure on-demand services and using Application Programming Interface (API) for interactions with hosts, switches, and routers, and the capability of adding new equipment in a simple and transparent manner. In general, the user does not manage the underlying hardware in the cloud infrastructure, but he controls the operating systems, storage and deployed applications. The service provider owns the equipment and is responsible for housing, running and maintaining it. The client typically pays on a per-use basis. Examples of IaaS include Amazon Elastic Cloud Computing (EC2), Amazon S3, GoGrid.



Fig. 2 Cloud Computing Service Delivery Models [26]

Combining the three types of clouds with the delivery models we get a holistic cloud illustration as seen in Figure 2, surrounded by connectivity devices coupled with information security themes. Virtualized physical resources, virtualized infrastructure, as well as virtualized middleware platforms and business applications are being provided and consumed as services in the Cloud [26]. Cloud vendors and clients' need to maintain Cloud computing security at all interfaces.

\section{CLOUD COMPUTING ARCHITECTURE: OVERVIEW}

Cloud computing can be divided into two sections, the user and the cloud. In most scenarios, the user is connected to the cloud via the internet. It is also possible for an organization to have a private cloud in which a user is connected via an intranet. However, both scenarios are identical other than the use of a private and public network or cloud [10]. The user sends requests to the cloud and the cloud provides the service. 


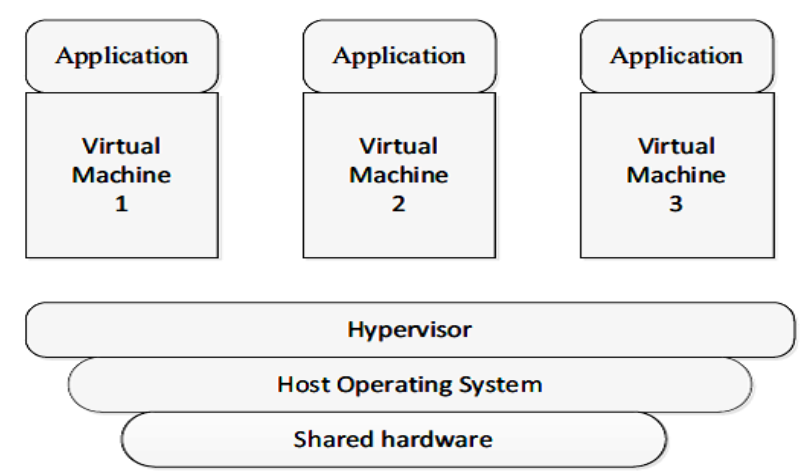

Fig. 3 Cloud Architecture [10]

Within the cloud, a central server is responsible for administering the system and in many ways functions as the operating system of the specific cloud network. Another name for this is called "middleware" which is the central server for a particular cloud. Examples include Google App Engine and Amazon EC2 [10].

\section{CLOUD COMPUTING ENTITIES}

Cloud providers and consumers are the two main entities in the business market. But, service brokers and resellers are the two more emerging service level entities in the Cloud world. These are discussed as follows

Cloud Providers: Includes Internet service providers, telecommunications companies, and large business process outsourcers that provide either the media (Internet connections) or infrastructure (hosted data centers) that enable consumers to access cloud services. Service providers may also include systems integrators that build and support data centers hosting private clouds and they offer different services (e.g., SaaS, PaaS, IaaS, and etc.) to the consumers, the service brokers or resellers [29].

Cloud Service Brokers: Includes technology consultants, business professional service organizations, registered brokers and agents, and influencers that help guide consumers in the selection of cloud computing solutions. Service brokers concentrate on the negotiation of the relationships between consumers and providers without owning or managing the whole Cloud infrastructure. Moreover, they add extra services on top of a Cloud provider's infrastructure to make up the user's Cloud environment.

Cloud Resellers: Resellers can become an important factor of the Cloud market when the Cloud providers will expand their business across continents. Cloud providers may choose local IT consultancy firms or resellers of their existing products to act as "resellers" for their Cloud-based products in a particular region. Cloud Consumers: End users belong to the category of Cloud consumers. However, also Cloud service brokers and resellers can belong to this category as soon as they are customers of another Cloud provider, broker or reseller.

\section{RESEARCH CHALLENGES IN CLOUD COMPUTING}

Cloud Computing research addresses the challenges of meeting the requirements of next generation private, public and hybrid cloud computing architectures, also the challenges of allowing applications and development platforms to take advantage of the benefits of cloud computing. The research on cloud computing is still at an early stage. Many existing issues have not been fully addressed, while new challenges keep emerging from industry applications. Some of the challenging research issues in cloud computing are given below [31, 41].

- Service Level Agreements (SLA's)

- Cloud Data Management \& Security

- Data Encryption

- Migration of virtual Machines

- Interoperability

- Access Controls

- Energy Management

- Multi-tenancy

- Server Consolidation

- Reliability \& Availability of Service

- Common Cloud Standards

- Platform Management 
Service Level Agreements (SLA's):Cloud is administrated by service level agreements that allow several instances of one application to be replicated on multiple servers if need arises; dependent on a priority scheme, the cloud may minimize or shut down a lower level application. A big challenge for the Cloud customers is to evaluate SLAs of Cloud vendors. Most vendors create SLAs to make a defensive shield against legal action, while offering minimal assurances to customers. So, there are some important issues, e.g., data protection, outages, and price structures that need to be taken into account by the customers before signing a contract with a provider [15]. The specification of SLAs will better reflect the customers' needs if they address the required issues at the right time. Some of the basic questions related to SLA are uptime i.e. are they going to be up $99.9 \%$ of the time or $99.99 \%$ of the time? And also how does that difference impact your ability to conduct the business? Is there any SLA associated with backup, archive, or preservation of data? If the service account becomes inactive then do they keep user data? If yes then how long?, So it's an important research area in cloud computing.

Cloud Data Management:Cloud data can be very large (e.g. text-based or scientific applications), unstructured or semi-structured, and typically append-only with rare updates Cloud data management an important research topic in cloud computing. Since service providers typically do not have access to the physical security system of data centers, they must rely on the infrastructure provider to achieve full data security. Even for a virtual private cloud, the service provider can only specify the security setting remotely, without knowing whether it is fully implemented. The infrastructure provider, in this context, must achieve the objectives like confidentiality, auditability. Confidentiality, for secure data access and transfer, and auditability, for attesting whether security setting of applications has been tampered or not. Confidentiality is usually achieved using cryptographic protocols, whereas auditability can be achieved using remote attestation techniques. However, in a virtualized environment like the clouds, VMs can dynamically migrate from one location to another; hence directly using remote attestation is not sufficient. In this case, it is critical to build trust mechanisms at every architectural layer of the cloud. Software frameworks such as MapReduce and its various implementations such as Hadoop are designed for distributed processing of data-intensive tasks; these frameworks typically operate on Internet-scale file systems such as GFS and HDFS. These file systems are different from traditional distributed file systems in their storage structure, access pattern and application programming interface. In particular, they do not implement the standard POSIX interface, and therefore introduce compatibility issues with legacy file systems and applications. Several research efforts have studied this problem [43].

Data Encryption:Encryption is a key technology for data security. Understand data in motion and data at rest encryption. Remember, security can range from simple (easy to manage, low cost and quite frankly, not very secure) all the way to highly secure (very complex, expensive to manage, and quite limiting in terms of access). You and the provider of your Cloud computing solution have many decisions and options to consider. For example, do the Web services APIs that you use to access the cloud, either programmatically, or with clients written to those APIs, provide SSL encryption for access, this is generally considered to be a standard. Once the object arrives at the cloud, it is decrypted, and stored. Is there an option to encrypt it prior to storing? Do you want to worry about encryption before you upload the file for cloud computing or do you prefer that the cloud computing service automatically do it for you? These are options, understand your cloud computing solution and make your decisions based on desired levels of security.

Migration of Virtual Machines:Applications are not hardware specific; various programs may run on one machine using virtualization or many machines may run one program. Virtualization can provide significant benefits in cloud computing by enabling virtual machine migration to balance load across the data center. In addition, virtual machine migration enables robust and highly responsive provisioning in data centers. Virtual machine migration has evolved from process migration techniques. More recently, Xen and VMWare have implemented "live" migration of VMs that involves extremely short downtimes ranging from tens of milliseconds to a second. The major benefit of VM migration is to avoid hotspots; however, this is not straightforward. Currently, detecting workload hotspots and initiating a migration lacks the agility to respond to sudden workload changes. Moreover, the in memory state should be transferred consistently and efficiently, with integrated consideration of resources for applications and physical servers [5].

Interoperability: This is the ability of two or more systems work together in order to exchange information and use that exchanged information. Many public cloud networks are configured as closed systems and are not designed to interact with each other. The lack of integration between these networks makes it difficult for organizations to combine their IT systems in the cloud and realize productivity gains and cost savings. To overcome this challenge, industry standards must be developed to help cloud service providers design interoperable platforms and enable data portability. Organizations need to automatically provision services, manage VM instances, and work with both cloud-based and enterprise-based applications using a single tool set that can function across existing programs and multiple cloud providers. In this case, there is a need to have 
cloud interoperability. Efforts are under way to solve this problem. For example, the Open Grid Forum, an industry group, is working on the Open Cloud Computing Interface, which would provide an API for managing different cloud platforms. Until now it has remained a challenging task in cloud computing.

Access Controls: Authentication and identity management is more important than ever. And, it is not really all that different. What level of enforcement of password strength and change frequency does the service provider invoke? What is the recovery methodology for password and account name? How are passwords delivered to users upon a change? What about logs and the ability to audit access? This is not all that different from how you secure your internal systems and data, and it works the same way, if you use strong passwords, changed frequently, with typical IT security processes, you will protect that element of access.

Energy Resource Management: Significant saving in the energy of a cloud data center without sacrificing SLA are an excellent economic incentive for data center operators and would also make a significant contribution to greater environmental sustainability. It has been estimated that the cost of powering and cooling accounts for $53 \%$ of the total operational expenditure of data centers. The goal is not only to cut down energy cost in data centers, but also to meet government regulations and environmental standards. Designing energy-efficient data centers has recently received considerable attention. This problem can be approached from several directions. For example, energy efficient hardware architecture that enables slowing down CPU speeds and turning off partial hardware components has become commonplace. Energy-aware job scheduling and server consolidation are two other ways to reduce power consumption by turning off unused machines. Recent research has also begun to study energy-efficient network protocols and infrastructures. A key challenge in all the above methods is to achieve a good trade-off between energy savings and application performance. In this respect, few researchers have recently started to investigate coordinated solutions for performance and power management in a dynamic cloud environment. The Global Energy Management Center(GEMC) can help companies monitor energy consumption patterns from multiple sources. These patterns can be further analyzed for usage, cost, and carbon footprint in a number of ways that help in optimizing energy. The center is uniquely positioned to service the clients across the globe by deploying a Remote Control Unit that has the capabilities to communicate to a cloud-based architecture [13].

Multi-tenancy:There are multiple types of cloud applications that users can access through the Internet, from small Internet-based widgets to large enterprise software applications that have increased security requirements based on the type of data being stored on the software vendor's infrastructure. These application requests require multi-tenancy for many reasons, the most important is cost. Multiple customers accessing the same hardware, application servers, and databases may affect response times and performance for other customers. For application-layer multi-tenancy specifically, resources are shared at each infrastructure layer and have valid security and performance concerns. For example, multiple service requests accessing resources at the same time increase wait times but not necessarily CPU time, or the number of connections to an HTTP server has been exhausted, and the service must wait until it can use an available connection or-in a worst-case scenariodrops the service request [39].

Server Consolidation:The increased resource utilization and reduction in power and cooling requirements achieved by server consolidation are now being expanded into the cloud. Server consolidation is an effective approach to maximize resource utilization while minimizing energy consumption in a cloud computing environment. Live VM migration technology is often used to consolidate VMs residing on multiple underutilized servers onto a single server, so that the remaining servers can be set to an energy-saving state. The problem of optimally consolidating servers in a data center is often formulated as a variant of the vector binpacking problem, which is an NP-hard optimization problem. Various heuristics have been proposed for this problem. Additionally, dependencies among VMs, such as communication requirements, have also been considered recently. However, server consolidation activities should not hurt application performance. It is known that the resource usage (also known as the footprint) of individual VMs may vary over time. For server resources that are shared among VMs, such as bandwidth, memory cache and disk I/O, maximally consolidating a server may result in resource congestion when a VM changes its footprint on the server. Hence, it is sometimes important to observe the fluctuations of VM footprints and use this information for effective server consolidation. Finally, the system must quickly react to resource congestions when they occur.

Reliability \& Availability of Service: The challenge of reliability comes into the picture when a cloud provider delivers on-demand software as a service. The software needs to have a reliability quality factor so that users can access it under any network conditions (such as during slow network connections). There are a few cases identified due to the unreliability of on-demand software. One of the examples is Apple's MobileMe cloud service, which stores and synchronizes data across multiple devices. It began with an embarrassing start when many users were not able to access mail and synchronize data correctly. To avoid such problems, providers are turning to technologies such as Google Gears, Adobe AIR, and Curl, which allow cloud based applications to 
run locally, some even allow them to run in the absence of a network connection. These tools give web applications access to the storage and processing capabilities of the desktop, forming a bridge between the cloud and the user's own computer. Considering the use of software such as 3D gaming applications and video conferencing systems, reliability is still a challenge to achieve for an IT solution that is based on cloud computing [41].

Common Cloud Standards:Security based accreditation for Cloud Computing would cover three main areas which are technology, personnel and operations. Technical standards are likely to be driven by organizations, such as, Jericho Forum1 before being ratified by established bodies, e.g., ISO2 (International Standard Organization). On the personnel side, the Institute for Information Security Professionals3 (IISP) is already offering formal accreditation for the security professionals. For the operational elements, there are some workable solutions such as tweaking the ISO 27001 and using it as the default measurement standard within the framework of the SAS 704. Currently, one of the main problems is that there are many fragmented activities going in the direction of Cloud accreditation, but a common body for the coordination of those activities is missing. The creation of a unified accreditation body to certify the Cloud services would also be a big challenge [15].

Platform Management:Challenges in delivering middleware capabilities for building, deploying, integrating and managing applications in a multi-tenant, elastic and scalable environments. One of the most important parts of cloud platforms provide various kind of platform for developers to write applications that run in the cloud, or use services provided from the cloud, or both. Different names are used for this kind of platform today, including on-demand platform and platform as a service (PaaS). This new way of supporting applications has great potential. When a development team creates an on-premises application (i.e., one that will run within an organization), much of what that application needs already exists. An operating system provides basic support for executing the application, interacting with storage, and more, while other computers in the environment offer services such as remote storage.

\section{CONCLUSION}

Cloud Computing, envisioned as the next generation architecture of IT Enterprise is a talk of the town these days. The way cloud has been dominating the IT market, a major shift towards the cloud can be expected in the coming years. Cloud computing offers real benefits to companies seeking a competitive edge in today's economy. Many more providers are moving into this area, and the competition is driving prices even lower. Attractive pricing, the ability to free up staff for other duties, and the ability to pay for "as needed" services will continue to drive more businesses to consider cloud computing. Mobile cloud computing is expected to emerge as one of the biggest market for cloud service providers and cloud developers.

Although Cloud computing can be seen as a new phenomenon which is set to revolutionize theway we use the Internet, there is much to be cautious about. There are many new technologiesemerging at a rapid rate, each with technological advancements and with the potential of makinghuman's lives easier. However, one must be very careful to understand the security risks andchallenges posed in utilizing these technologies. Cloud computing is no exception.Cloud service providers need to inform their customers on the level of security that they provide on their cloud.This research effort presents an overview of Cloud Computing, building blocks of Cloud Computing which includes different models of cloud computing, overview of Cloud Computing architecture and Cloud Computing entities. Furthermore, research challenges which are currently faced in the Cloud computing were alsohighlighted.

Cloud computing has the potential to become a frontrunner in promoting a secure,virtual and economically viable IT solution in the future. As the development of cloud computing technology is still at an early stage, this research effort will provide a better understanding of the design challenges of cloud computing, and pave the way for further research in this area.

\section{REFERENCES}

[1] A Platform Computing Whitepaper. "Enterprise Cloud Computing: Transforming IT.” Platform Computing, pp6, 2010.

[2] B.P. Rimal, Choi Eunmi, I. Lumb, "A Taxonomy and Survey of Cloud Computing Systems", Intl. Joint Conference on INC, IMS and IDC, 2009, pp. 44-51,Seoul, Aug, 2009. DOI: 10.1109/NCM.2009.218

[3] B. R. Kandukuri, R. Paturi V, A. Rakshit, "Cloud Security Issues", In Proceedings of IEEE International Conference on Services Computing, pp. 517-520, 2009.

[4] Cloud Computing. Wikipdia. Available at http://en.wikipedia.org/wiki/Cloud_computing

[5] Cong Wang, Qian Wang, KuiRen, and Wenjing Lou, "Ensuring Data Storage Security in Cloud Computing," 17 th International workshop on Quality of Service, USA, pp.1-9, July 13-15, 2009, ISBN: 978-1-4244-3875-4

[6] C. Weinhardt, A. Anandasivam, B. Blau, and J. Stosser."Business Models in the Service World.”IT Professional, vol. 11, pp. 28-33, 2009.

[7] Daniel Oliveira and Eduardo Ogasawara. Article: Is Cloud Computing the Solution for Brazilian Researchers?. International Journal of Computer Applications 6(8):19-23, September 2010. 
[8] D. Oliveira, F. Baião, and M. Mattoso, 2010, "Towards Taxonomy for Cloud Computing from an e-Science Perspective", Cloud Computing: Principles, Systems and Applications (to be published), Heidelberg: Springer-Verlag.

[9] Dr. Gurdev Singh, ShanuSood, Amit Sharma, "CM- Measurement Facets for Cloud Performance", IJCA, , Lecturer, Computer science \& Engineering, Eternal University, Baru Sahib (India), Volume 23 No.3, June 2011.

[10] Ertaul, L. and Singhal, S. 2009. Security Challenges in Cloud Computing. California State University, East Bay. Academic paper http://www.mcs.csueastbay.edu/ lertaul/Cloud\%20Security\%20CamREADY.pdf

[11] Gaoyun Chen, Jun Lu and Jian Huang, Zexu Wu, "SaaAS - The Mobile Agent based Service for Cloud Computing in Internet Environment", Sixth International Conference on Natural Computation, ICNC 2010, pp. 2935-2939, IEEE, Yantai, Shandong,China, 2010. ISBN: 978-1-4244-5958-2.

[12] Global Netoptex Incorporated. "Demystifying the cloud.Important opportunities, crucial choices." pp4-14. Available: http://www.gni.com [Dec. 13, 2009].

[13] Hanqian Wu, Yi Ding, Winer, C., Li Yao, "Network Security for Virtual Machines in Cloud Computing," 5th Int'l Conference on Computer Sciences and Convergence Information Technology, pp. 18-21, Seoul, Nov. 30- Dec. 2, 2010. ISBN: 978-1-4244-8567-3.

[14] Harjit Singh Lamba and Gurdev Singh, "Cloud Computing-Future Framework for e-management of NGO's", IJoAT, ISSN 09764860, Vol 2, No 3, Department Of Computer Science, Eternal University, Baru Sahib, HP, India, July 2011.

[15] Harold C. Lin, ShivnathBabu, Jeffrey S. Chase, Sujay S. Parekh, "Automated Control in Cloud Computing: Opportunities and Challenges", Proc. of the $1^{\text {st }}$ Workshop on Automated control for data centres and clouds, New York, NY, USA, pp. 13-18, 2009, ISBN: 978-1-60558-585-7.

[16] Hoang T. Dinh, Chonho Lee, DusitNiyato, Ping Wang, "A Survey of Mobile Cloud Computing: Architecture, Applications and Approaches", Wireless Communications and Mobile Computing, Wiley Inc.

[17] I. Foster, Y. Zhao, I. Raicu, and S. Lu, 2008, Cloud Computing and Grid Computing 360-Degree Compared, In: Grid Computing Environments Workshop, 2008. GCE '08, p. 10,1.

[18] Joachim Schaper, 2010, "Cloud Services", 4th IEEE International Conference on DEST, Germany.

[19] K.Mukherjee and G.Sahoo. Article: Cloud Computing: Future Framework for e-Governance. International Journalof Computer Applications 7(7):31-34, October.

[20] Kuyoro S.O., Ibikunle F., Awodele O., “Cloud Computing Security Issues \& Challenges”, IJCN, Vol. 3 Issue 5: 2011, pp. 247-255.

[21] Liladhar R Rewatkar and Ujwal A Lanjewar. Article: Implementation of Cloud Computing on Web Application. International Journal of Computer Applications 2(8):28-32, June 2010. Published By Foundation of Computer Science.

[22] L.M. Vaquero, L. Rodero-Merino, J. Caceres, and M. Lindner, 2009, A break in the clouds: towards a cloud definition, SIGCOMM Comput. Commun. Rev., v. 39, n. 1, p. 50-55

[23] L. Wang, GregorLaszewski, Marcel Kunze, Jie Tao, "Cloud Computing: A Perspective Study", New Generation ComputingAdvances of Distributed Information Processing, pp. 137-146, vol. 28, no. 2, 2008. DOI: 10.1007/s00354-008-0081-5

[24] Meiko Jensen, JorgSchwenk, Nils Gruschka, Luigi Lo Iacon, "On technical Security Issues in Cloud Computing”, Proc. of IEEE International Conference on Cloud Computing (CLOUD-II, 2009), pp. 109-116, India, 2009.

[25] Muzafar Ahmad Bhat, Razeef Mohd Shah, Bashir Ahmad and InayatRasoolBhat, "Cloud Computing: A Solution to Information Support Systems", IJCA, Vol. 11-No.5, December 2010, pp. 5-9.

[26] M. Klems, A. Lenk, J. Nimis, T. Sandholm and S. Tai. "What's Inside the Cloud? An Architectural Map of the Cloud Landscape."IEEEXplore, pp 23-31, Jun. 2009.

[27] Peter Mell, Timothy Grance, "The NIST Definition of Cloud Computing”, Jan, 2011. http://docs.ismgcorp.com/files/external/DraftSP-800-145_cloud-definition.pdf

[28] Prince Jain, "Security Issues \& their solution in Cloud Computing”, International Journal of Computing \& Business Research.

[29] Pring et al., "Forecast: Sizing the cloud; understanding the opportunities in cloud services," Gartner Inc., Tech. Rep. G00166525, March 2009 .

[30] Problems Faced by Cloud Computing, Lord CrusAd3r,dl.packetstormsecurity.net/.../ProblemsFacedbyCloudComputing.pdf.

[31] Rabi Prasad Padhy, ManasRajanPatra and Suresh Chandra Satapathy, "Cloud Computing: Security Issues \& Research Challenges", IJCSITS, Vol. 1-No.2, December 2011, pp. 136-146.

[32] RohitBhadauria, SugataSanyal, "Survey on Security Issues in Cloud Computing \& Associated Mitigation Techniques", IJCA, Vol. 47-No. 18, June 2012, pp. 47-66.

[33] R. L Grossman, “The Case for Cloud Computing,” IT Professional, vol. 11(2), pp. 23-27, 2009, ISSN: 1520-9202.

[34] R. Maggiani, Communication Consultant, Solari Communication, "Cloud Computing is Changing How we Communicate", 2009 IEEE International Professional Conference, IPCC, pp. 1-4, Waikiki, HI, USA, July 19- 22, 2009. ISBN: 978-1-4244-4357-4.

[35] SangeetaSen, RituparnaChaki, "Handling Write Lock Assignment in Cloud Computing Environment", Communications in Computer and Information Science, vol. 245, issue. 7, pp. 221-230, 2011. DOI: 10.1007/978-3-642-27245-5_27

[36] SonalAnand, Sarvesh Gupta, ShwetaFatnani, Varsha Sharma and Deepti Jain. Article: Semantic Cloud for Mobile Technology. International Journal of Computer Applications 8(12):1-4, October 2010.

[37] Sun Microsystems White Paper, - Introduction to Cloud Computing Architecture, IJ June 2009.

[38] S. Arnold (2009, Jul.). "Cloud computing and the issue of privacy.”KM World, pp14-22. Available: www.kmworld.com [Aug. 19, 2009].

[39] S. Subashini, V. Kavitha, "A survey on security issues in service delivery models of cloud computing"; Journal of Network and Computer Applications, Vol. 34(1), pp 1-11, Academic Press Ltd., UK, 2011, ISSN: 1084-8045.

[40] Timothy Wood, PrashantShenoy, Alexandre Gerber, K.K. Ramkrishnan, Jacobus Van der Merwe, "The Case for Enterprise-Ready Virtual Private Clouds", HotCloud'09 Proceedings of the 2009 conference on Hot topics in cloud computing, San Diego, CA, USA, 2009. http://www.usenix.org/event/hotcloud09/tech/full_papers/wood.pdf

[41] V. Krishna Reddy, B. ThirumalRao, Dr. L.S.S. Reddy, P.SaiKiran "Research Issues in Cloud Computing " Global Journal of Computer Science and Technology, Volume 11, Issue 11, July 2011.

[42] W.K. Chan, Lijun Mei, and Zhenyu Zhang, "Modeling and testing of cloud applications", to appear in Proceedings of2009 IEEE Asia-Pacific Services Computing Conference (APSCC 2009), (Singapore, December 7-11, 2009), IEEE Computer Society Press, Los Alamitos, CA, USA, 2009

[43] X. Zhang, N. Wuwong, H. Li, and X. J. Zhang, "Information Security Risk Management Framework for the Cloud Computing Environments", In Proceedings of 10th IEEE International Conference on Computer and Information Technology, pp. 1328- 1334, 2010. 